\title{
Milliequivalent per Millimole
}

National Cancer Institute

\section{Source}

National Cancer Institute. Milliequivalent per Millimole. NCI Thesaurus. Code C92616.

A concentration unit measured as a number of one thousandth of an equivalent weight per millimole of substance. 